\title{
A charge of the erythrocyte test by automated method
}

\author{
Oksana Gutsul $^{1}$, Nikolay Shaplavsky ${ }^{1}$, Vasiliy Buzhdygan ${ }^{2}$, Vsevolod Slobodian $^{3}$ \\ ${ }^{1}$ Department of Biological Physics and Medical Informatics, Bukovinian State Medical University, Chernivtsi, Ukraine \\ ${ }^{2}$ Chernivtsi Regional Hospital, Chernivtsi, Ukraine \\ ${ }^{3}$ Department of Electronics and Electrotechnics, Yuriy Fedkovych Chernivtsi National University, Chernivtsi, Ukraine \\ Email: oksana.v.s@mail.ru
}

Received 7 November 2011; revised 6 January 2012; accepted 29 January 2012

\begin{abstract}
New methods of an automated evaluation of the blood rheological parameters: 1) the viscosity, 2) electric conductivity and 3) the charge of erythrocytes have been theoretically and experimentally substantiated by way of registering the $Q$-factor of the tuned-circuit, containing a capillary with the blood flow. A design of an electronic measuring complex is based on modeling the physical blood conditions in the natural environment.
\end{abstract}

Keywords: Biophysics; Physical Methods of Evaluating Blood Rheological Properties and Parameters; Quality Factor (Q-Factor); Designing

\section{INTRODUCTION}

A foreign material contacting with a living tissue, for example with blood immediately brings about numerous physical changes in it. It is precisely this fact that explains why biometry of the physical parameters of blood or its components in the natural environment remains highly doubtful. Thus, a study of electrophoretic mobility, blood electric conductivity in contact with electrodes, its viscosity, when being in touch with the material of viscosimeters (the thermorheologic effect [1]) or the surface potential of erythrocytes under such conditions with the use of modern medical equipment for physical analyses have found no effective application [2]. Meanwhile, it was corroborated long ago that the question is about the principal biophysical properties of blood owing to which its capacity for microcirculation in the natural environment (in vivo) [2]. The problem of erroneous technical decisions, in particular, the methods of a blood analysis listed above lies in the fact that in vitro (in an artificial environment) they do not simulate in vivo physicochemical conditions.

However, in order to approximate to such an adequate model it is necessary, at the minimum, to take as an axiom that friction in the bloodstream and intercellular communications, in a contact measured by hectares, is insignificant and is determined by the Q-factor of the cardiovascular complex in case of transformations of different types of energy. It is also necessary to believe in the theoretical and experimental proofs of the adaptive dynamics of the erythrocytes' charge, the dynamics implementing the mechanism of the blood microcirculation [2].

But in spite of the fact that such proofs have already been mentioned for many decades in scientific literature [2-4], the proofs corresponding to the laws of the thermodynamics of biological systems, the informational layer of the biological transport (energy and mass transfer) remains one of the most topical in the fundamental researches of physics, molecular biology and medicine.

Within the limits of this paper we confine ourselves to the postulates stated, forming the basis of the design of an electronic measuring complex for blood biometry.

\section{MATERIALS AND METHODS}

\section{Design Methodology}

In order to exclude intensive physical effects on the blood system (friction, static electricity), a measurement of the viscosity, electric conductivity of blood and the charge of the blood erythrocytes has been performed via registering electromagnetic losses of this tissue (Qfactor), proceeding in a capillary solenoid, associated with the oscillatory circuit by induction, i.e. outside a mechanical contact. The capillary solenoid itself is made of Teflon - a hydrophobic (water-repelling) material called bioinert, which, when contacted, does not essentially change the blood function [2].

The design of the measuring complex and the principle of its action are explained in Figure 1 and Figure 2.

For the first time here registering the dynamics of the Q-factor is an indicator of the velocity of the blood flow and its electrical conductivity. A change of the Q-factor appears on the monitor upon the entry of examined blood (or plasma) into a capillary after the control solution 


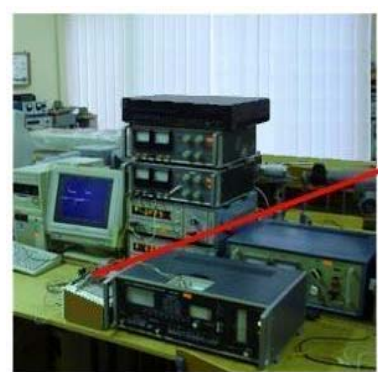

(a)

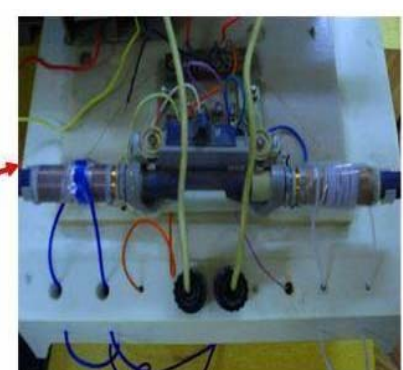

(b)
Figure 1. A measuring complex to determine the blood viscosity (a); a capillary solenoid is connected by induction with an oscillatory circuit of the Q-meter (b).

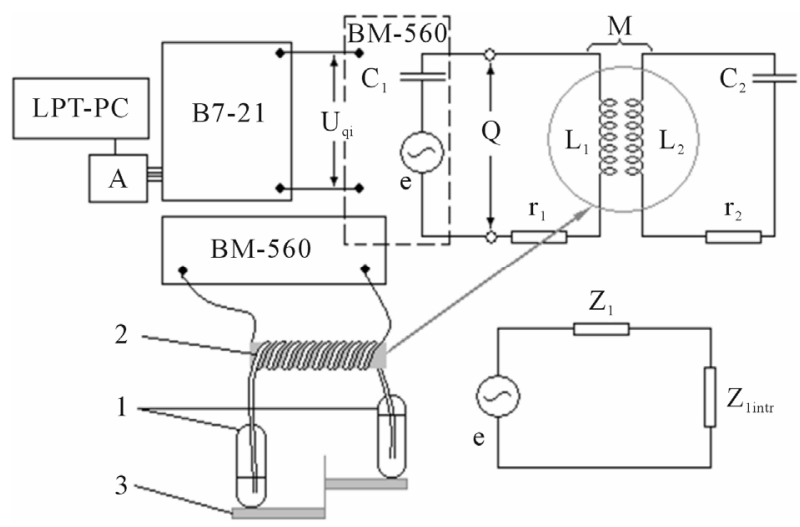

Figure 2. An electric and equivalent diagram of coupled circuits. 1-capsules with fluids under study; 2-a capillary solenoid; 3-a micrometric regulator of the level of capsules; $B 7-21-$ a digital voltmeter; $A$ - an interface adapter to a personal computer LPT-PC; $B M-560-\mathrm{Q}$ meter; $C_{1}$ and $C_{2}$ - the capacity of the first and second oscillatory circuits; $L_{1}$ and $L_{2}$ - the inductance circuits; $Q$-the Q-factor of an equivalent circuit; $U q_{i}$ - the voltage defined by the Q-factor (the output voltage of the BM-560 device); $M$ - the natural inductance factor; $r_{1}$ and $r_{2}$ : the active resistance of circuits; $e$ - the source amplitude of the electromotive force. $Z_{1}$ and $Z_{\text {intr }}$-introduced and own complex impedances of an equivalent circuit.

(Figure 3) and evidences about the velocity of its flow, whereas the level of its Q-factor points out electric conductivity.

A complete theoretical and experimental substantiation of the new method was submitted by us earlier [5], where on the basis of the laws of radio electronics and hydrodynamics we have deduced a formula according to which a computer program calculates viscosity automatically:

$$
\eta=K \frac{\Delta p}{v_{c}} .
$$

where $\eta$-viscosity; $K$ - the calibrating constant of the measuring complex; $\Delta p-$ a difference of the pressures of the levels of fluids in the capsules (Figure 2); $v_{c}$ - the effective velocity of the movement of fluids in the

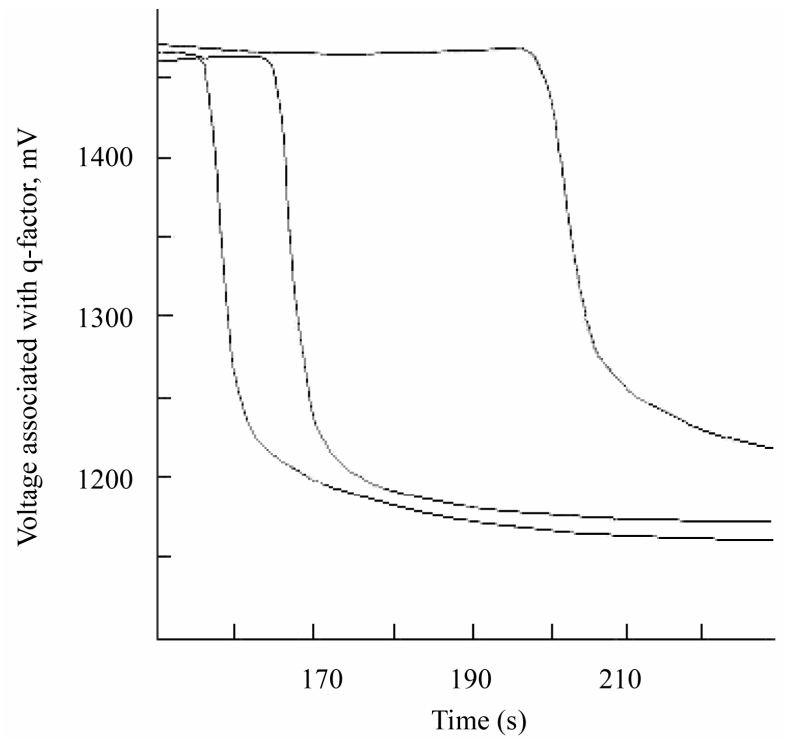

Figure 3. Registration of a Q-factor change of the oscillatory circuit with the plasma flow of different blood groups.

capillary. Thus, for the first time we succeeded in detecting the genetic spectrum of the blood viscosity and revealing the efficiency of the new physical analysis [6].

An elementary dependence of the Q-factor on electric conductivity introduced into the program enables to obtain findings of the blood and plasma electric conductivity outside a mechanical contact with electrodes [7].

\section{RESULTS}

The difference of this index in whole blood and its plasma upon introducing into the program of the findings of clinical blood studies makes it possible to automatically compute the natural charge of erythrocytes which has been determined for the first time according to the deduced formula:

$$
|e| Z_{e r}=-\left[\sigma_{b l}-(1-k) \sigma_{p l}\right] / n_{e r} \mu^{+} .
$$

where $|e| Z_{e r}$-is an absolute charge of an erythrocyte (Coulomb, $C$ ); $\sigma_{b l}$ the blood electric conductivity; $\sigma_{p l}$ - the blood plasma electric conductivity; $k$ - the index of hematocrit (a ratio of the volume of the blood erythrocytes and whole blood); $n_{e r}$ - the content of erythrocytes; $\mu^{+}$- the mobility of the principal plasmatic cations $\left(\mathrm{Na}^{+}\right.$ and $\mathrm{K}^{+}$).

It appears that the charge of an erythrocyte normally makes up

$$
|e| Z_{e r}=-3.19 \times 10^{-10} \mathrm{C}
$$

or in effective non-compensated electrons it adopts the following value

$$
Z_{e r}=-2.0 \times 10^{9} \mathrm{C} .
$$


To calculate the charge of erythrocytes output parameters are: pulse of $q$-factor whole blood and its plasma displays on the display when they flow through bioinert capillary (Figure 4).

To determine the charge of erythrocytes outside a mechanical contact with electrodes automated method used in a clinical laboratory blood $(4 \mathrm{ml})$, which was taken from ulnar vein and added heparin $(10 \mathrm{U} / \mathrm{ml})$. Plasma ( $1 \mathrm{ml})$ obtained by centrifugation at $3000 \mathrm{rev} / \mathrm{min}$ for 15 minutes. Feed the clinical data of blood erythrocytes, hematocrit, the content of $\mathrm{Na}^{+}$and $\mathrm{K}^{+}$into the computer of measuring complex with bioinert capillary, where the Q-factor registered [5]. A capsule of distillated water placed at the standard levels of electrolytes difference $\Delta h=2.5 \mathrm{~cm}$ [5]. Capsule replaced by 20 seconds in a capsule of plasma after stabilization on the display of q-factor-level, and later (Figure 4) see to $\mathrm{q}$-factor impulse of plasma. A similar manipulation of repeated for blood (plasma which had just studied). Both values of Q-factor (in Figure 4 is shown Q-factor of blood and plasma) calculating of the program the erythrocyte charge in Coulomb.

So you can observe the effect of adrenaline on the average charge of erythrocytes. For example, in $1 \mathrm{ml}$ of blood added to drops of $\mathrm{NaCl}$ solution containing $166 \times$ $10^{-7}$ gram of adrenaline (in the control brought a drop of saline) during the merit of this solution (Figure 5) observed the phenomenon of loss of merit whole blood, increasing its electrical conductivity by due to mobile cations that have left due to dissociation of acidic groups array of red blood cells. In Figure 5 shows the superposition of the experiment and control. Thus, direct measurements confirmed before we found red blood cells increase the charge for the actions of catecholamine's in

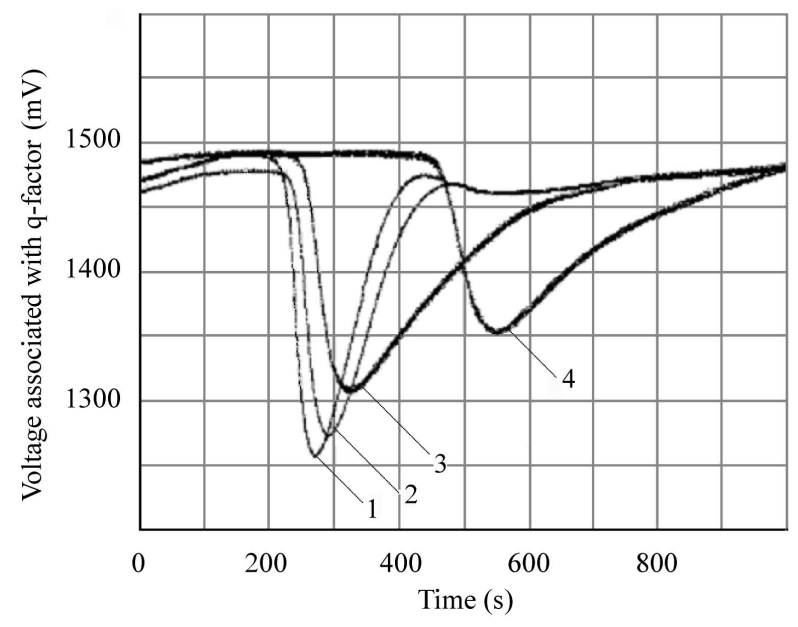

Figure 4. Dependence of high voltage associated with qfactor $(\mathrm{Uq}, \mathrm{mV})$ with plasma and blood flow $(\mathrm{t}, \mathrm{c})$ followed by distilled water: 1, 3-plasma and blood, respectively, after treatment of asthma; 2, 4-plasma and blood to treat asthma.

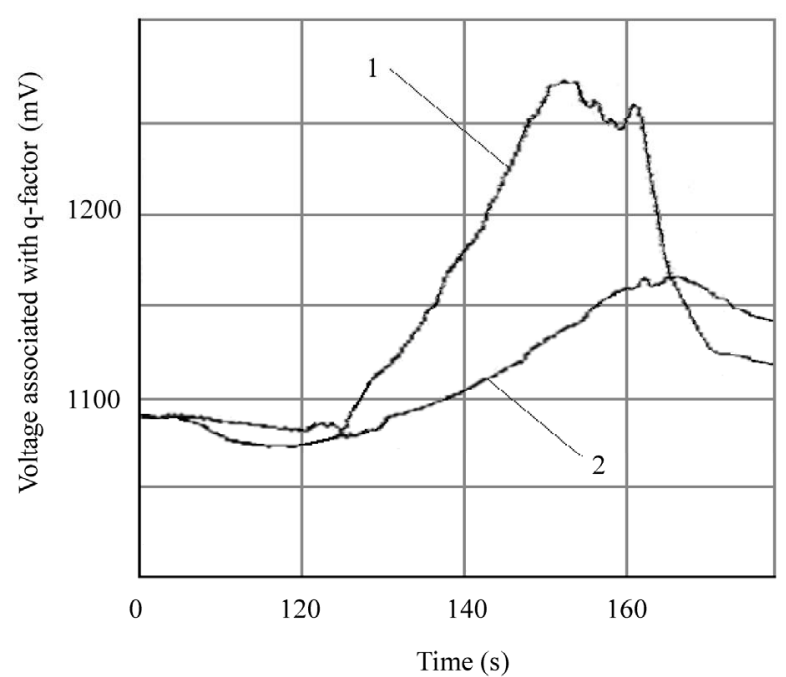

Figure 5. Dependence of stress associated with q-factor (Uq, $\mathrm{mV})$, since the flow of blood $(\mathrm{t}, \mathrm{s})$ followed by saline. 1blood; 2-blood for the actions of adrenaline.

bioinert conditions using indirect methods [2].

\section{CONCLUSIONS}

Fundamentally new method for automated determination of erythrocyte charge, allows you to:

- Find this option by direct measurement;

- In a model in vivo to identify adaptive changes in erythrocyte charge.

Thus, designing electronic equipment for biometry requires a reproduction of a model of the functioning tissue in the object-registration system. A measurement of the Q-factor of the living tissue in this case appears to be informative.

A suggested design has manifest prospects of its use in different spheres of production where, in particular, an evaluation of the viscosity of fluids often boils down not to a study of the properties of substances, but to establishing a consequence of their interrelation with the physically active medium of measuring devices [8].

\section{REFERENCES}

[1] Kuz'menko, O.Yu. (2003) Rotary viscometers with the superhigh frequency system of transformation of a controlled parameter. Author's abstract of dissert of Cand. of Med. Sci.. Tambov, TSTU. (In Russian). http://www.twirpx.com/file/510318/

[2] Shaplavs'kyi, M.V. (1996) Bioinertization as a biological function. Prut., Chernivtsi. (In Ukrainian).

[3] Fahraeus, R. and Linqvist, T. (1931) The viscosity of the blood in narrow capillary tubes. American Journal of Physiology, 96, 562-568.

[4] Isenberg, J. (1953) A note of the flow of blood in capillary tubes. Bulletin of Mathematical Biophysics, 15, 149- 
152. doi:10.1007/BF02476379

[5] Shaplavs'kyi, M.V., Pishak, V.P., Slobodian, O.V. and Hrygoryshyn, P.M. (2008) Patent 35766 of Ukraine. Electrode-free method for automated measurement of viscosity of biological fluids. Bulletin No. 19. (In Ukrainian).

http://base.ukrpatent.org/searchINV/search.php?action=vi ewdetails\&IdClaim $=124456$

[6] Gutsul, O.V., Buzhdygan, V.V., Shaplavs'kyi, M.V. and Grygoryshyn, P.M. (2010) The measuring complex for the viscosity test of the biological fluids. Buk. Med. Herald., 14, 158-164 (In Ukrainian).
[7] Shaplavs'kyi, M.V., Pishak, V.P., et al. (2008) Patent 36976 of Ukraine. Electrode-less method for automated measurement of specific resistance of electrolytes and biological liquids. Bulletin No. 21 (In Ukrainian).

http://base.ukrpatent.org/searchINV/search.php?action=vi ewdetails\&IdClaim $=125813$

[8] Yevdokimov, I.N. and Yelisieiev, N.Yu. (2005) Molecular mechanisms of the viscosity of a liquid and gas. Part I. Principal notions. RSU of Oil and Gas Named after I.M. Gubkin Press, Moscow (In Russian). http://www.twirpx.com/file/439320/ 